\title{
The Effect of Inhibitors of DNA Repair on the Genetic Instability of Streptomyces cattleya
}

\author{
By V. E. COYNE, K. USDIN AND R. KIRBY* \\ Department of Microbiology, University of Cape Town, Rondebosch 7700, Cape Town, \\ South Africa
}

(Received 11 May 1983; revised 2 September 1983)

\begin{abstract}
Various streptomycetes show well defined instabilities that do not appear to be attributable to plasmid loss. The unstable phenotype, in many cases, arises at frequencies too high to be explained by point mutations. The frequency of instability can be enhanced by UV irradiation. Two major repair systems have been found in Escherichia coli: the 'error-free' system which is inhibited by caffeine and the 'error-prone' system which is inhibited by arsenite. Using spores of Streptomyces cattleya NRRL 8057 and the virulent actinophage VC11 we have shown that a caffeine inhibitable, host mediated UV repair system is active in spores during early development. Some evidence was also found for the presence of an arsenite inhibitable UV repair system. The caffeine inhibitable UV repair system was found to be involved in the induction of genetic instability in $S$. cattleya. The arsenite system may be implicated in the repair of such events. Genetic instability was also induced by single strand breaks in DNA caused by ${ }^{32} \mathrm{P}$.
\end{abstract}

\section{INTRODUCTION}

Streptomyces cattleya produces the broad spectrum beta-lactamase resistant beta-lactam antibiotic thienomycin (Martin et al., 1979). A characteristic genetic instability which is associated with a change from an aerial mycelium producing phenotype $\left(\mathrm{Amy}^{+}\right)$to an aerial mycelium non-producing phenotype (Amy ${ }^{-}$) occurs at high frequency in $S$. cattleya and is almost always associated with a change from arginine prototrophy to arginine auxotrophy. The metabolic block is in the arginine biosynthetic pathway and involves a deficiency of the enzyme argininosuccinate synthetase. The instability is sometimes reversible and is also associated with other phenotypic changes such as in the growth rate and antibiotic production. Instability of this type is found in a number of Streptomyces species and could involve 'transposon-like' events. Unlike most cases of transposition in eubacteria, this instability is induced by acriflavine or UV light (Pogell, 1979; Kirby \& Lewis, 1981). The fact that UV light acts as an inducer of genetic instability in Streptomyces spp. suggests that UV repair systems may be involved in the genetic instability phenomenon.

The use of inhibitors of DNA repair to study UV repair systems in Escherichia coli is well established. Caffeine is thought to be a specific inhibitor of 'error-free' repair (Rothman, 1980). Sodium arsenite is an inhibitor of 'error-prone' rec $A$ dependent repair (Rossman et al., 1975). No recA mutants have been isolated in Streptomyces but many Streptomyces species exhibit a high level of recombination which may indicate the presence of a somewhat similar recombination system.

A major problem associated with the use of inhibitors of UV repair is the separation of pleiotropic effects of the chemicals. To determine whether caffeine and arsenite are acting at the level of DNA repair it is necessary to use a bacteriophage which can be subjected to UV irradiation independently of the host organism and the chemicals. 
Most of the studies of genetic instability in Streptomyces spp. have used spores as the starting point for the work. The mycelial nature of vegetative cells precludes working with unicellular entities whereas Streptomyces spores are thought in general to be unigenomic. It was for this reason that spores were used in this study. There are, however, two disadvantages: firstly, the repair system in vegetative cells and spores may be different; and secondly, when using bacteriophage there will be a time delay of about $6 \mathrm{~h}$ between irradiation and entry of the bacteriophage DNA to the germinating spore. In the former case the difference is probably not too important as most previous studies have been done on spores. The latter problem is probably more important as some repair systems are only induced for a short time and will have decayed before entry of the bacteriophage DNA.

\section{METHODS}

Bacteria and bacteriophage. The $S$. cattleya strain used was NRRL 8057 . The bacteriophage used was a broad host range, virulent actinophage VC11 isolated from the soil of Table Mountain, Cape Town, South Africa. The handling of the organisms was as described in Flett et al. (1979).

Media and reagents. Streptomyces cattleya was grown on Malt-3 agar (Kirby \& Lewis, 1981). Propagation of the phage was on nutrient broth agar and soft nutrient broth agar. Spore dilutions were made in sterile distilled water and phage dilutions were made in nutrient broth (Flett et al., 1979).

UV irradiation. This was done at $254 \mathrm{~nm}$ using an English Electric G8T5 tube which provided an irradiation level of $5 \mathrm{~J} \mathrm{~m}^{-2}$. All irradiation measurements were made with a UV meter model 225 (Ultra-violet Products, San Gabriel, Calif., USA). The volume irradiated was $5 \mathrm{ml}$ in a $9 \mathrm{~cm}$ Petri dish with mixing every $10 \mathrm{~s}$.

Identification of $\mathrm{Amy}^{-} \mathrm{Arg}^{-}$variants. These were identified visually after $7 \mathrm{~d}$ incubation and a proportion were checked for their auxotrophic requirements.

Effect of ${ }^{32} \mathrm{P}$ on the induction of $\mathrm{Amy}^{-} \mathrm{Arg}^{-}$variants. ${ }^{32} \mathrm{P}$-labelled orthophosphate $(0.4 \mathrm{mCi}, 14.8 \mathrm{MBq})$ was added to a slant of M-3 agar which was then inoculated with a spore suspension of $S$. cattleya and incubated for $18 \mathrm{~d}$. The spores were then harvested, washed, stored at $-70^{\circ} \mathrm{C}$ for one half-life and plated on M-3 agar. After sporulation the frequency of $\mathrm{Amy}^{-} \mathrm{Arg}^{-}$variants was determined.

\section{RESULTS}

\section{Occurrence of Amy ${ }^{-}$variants of S. cattleya and their phenotype}

Amy $^{-}$variants of $S$. cattleya occur both spontaneously and after UV treatment. Of the spontaneously occurring $\mathrm{Amy}^{-}$variants, $56 \%$ were $\mathrm{Arg}^{-}$with a block in arginine biosynthesis at argininosuccinate synthetase; $11 \%$ were $\mathrm{Arg}^{-} \mathrm{Met}^{-} ; 5 \%$ were $\mathrm{Met}^{-} ; 17 \%$ were prototrophic and the remainder had other auxotrophic requirements. UV irradiation $\left(150 \mathrm{~J} \mathrm{~m}^{-2}\right)$ of the spores gave Amy ${ }^{-}$clones of which $91 \%$ were $\mathrm{Arg}^{-}, 2 \%$ were $\mathrm{Met}^{-}$and $1 \%$ were $\mathrm{Arg}^{-} \mathrm{Met}^{-}$. Figure 1 shows the common Amy- phenotypes.

\section{$U V$ irradiation repair in the spores of $S$. cattleya}

The presence of a photoreactivation and/or a liquid holding recovery in $S$. cattleya would have affected the survival of both spores and bacteriophage during these experiments. Spores were irradiated to a level of $50 \mathrm{~J} \mathrm{~m}^{-2}$ in the dark and then held either in the dark or in sunlight for up to $270 \mathrm{~min}$ in sterile distilled water. As no increase in spore survival was detected in either case, no liquid holding recovery or photoreactivation was in action over this period. Therefore, all subsequent experiments were done within this time period. The absence of these two systems is not unusual in Streptomyces spp.; the majority seem to be like $S$. cattleya.

The concentration of the inhibitors of UV repair used was estimated by a method similar to that of Schumann et al. (1982). Spores of S. cattleya $(0 \cdot 1 \mathrm{ml})$ were spotted on to M-3 agar plates containing increasing concentrations of the inhibitors in order to determine the MIC. An MIC of $3 \mathrm{mg} \mathrm{ml}^{-1}$ was obtained for caffeine and $0.45 \mathrm{mg} \mathrm{ml}^{-1}$ for sodium arsenite. Further experiments were done at one-third of the MIC for these inhibitors. At this concentration the inhibitors reduced the viability of $S$. cattleya spores by only $10 \%$. The results of subsequent experiments have been corrected for this inhibitory effect (determined from controls).

A direct study of spore survival after UV irradiation gives an indication of whether there are $U V$ repair systems active in spores during germination. A decrease in spore viability after UV 

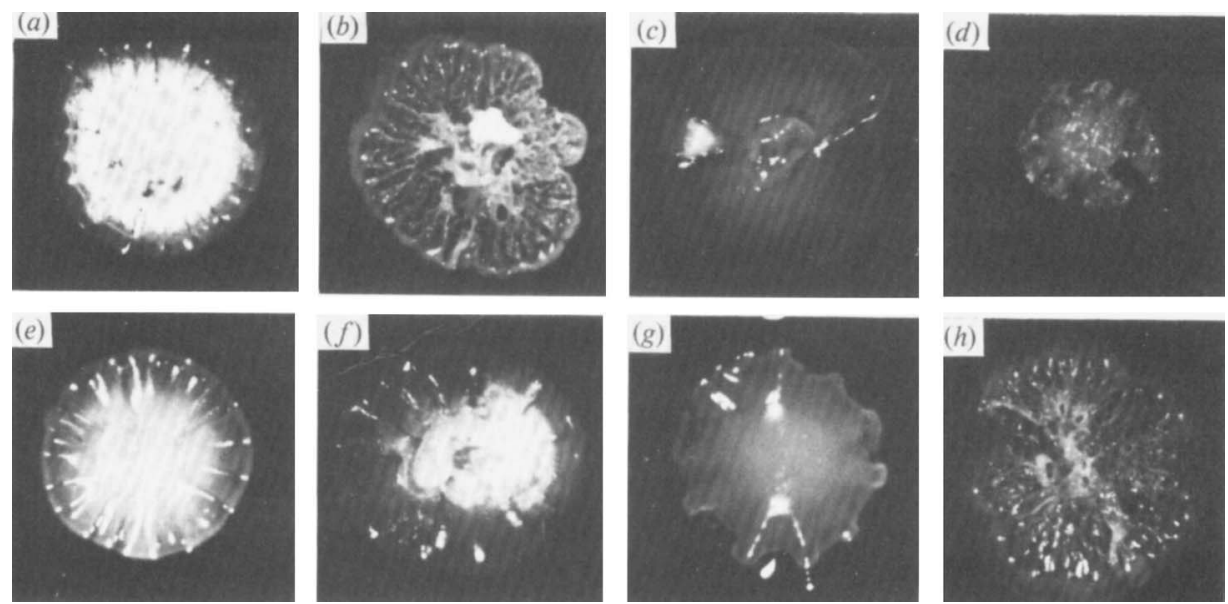

Fig. 1. Phenotypes of common Amy- mutants. (a) Wild type; $(b)-(h)$ Amy- mutants.

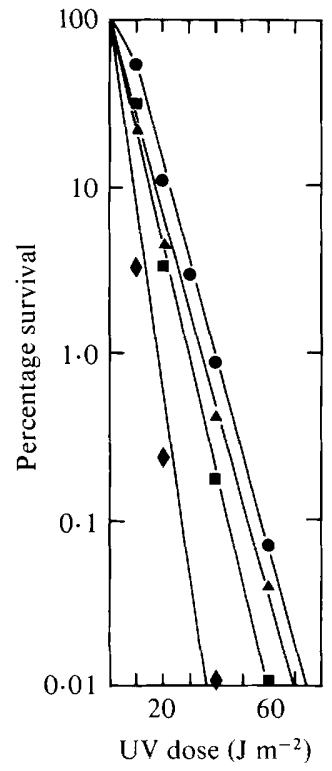

Fig. 2

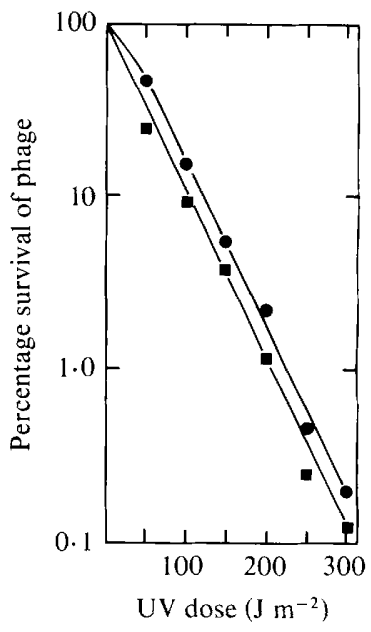

Fig. 3

Fig. 2. Effect of caffeine and sodium arsenite on the survival of Streptomyces cattleya spores after UV irradiation. $\boldsymbol{-}$, No inhibitor; $\mathbf{D},+$ caffeine; $\boldsymbol{\Delta},+$ sodium arsenite; $\bullet+$ caffeine and sodium arsenite.

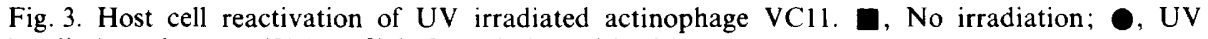
irradiation of spores $\left(50 \mathrm{~J} \mathrm{~m}^{-2}\right)$ before plating with phage.

irradiation and germination in the presence of an inhibitor does not prove the presence of a repair system because an interaction between the inhibitors and UV irradiation could cause a viability variation which would mimic the response. The presence of a shoulder in the UV survival curve and the reduction in survival after UV irradiation in the presence of either inhibitor showed that UV repair systems may have been active (Fig. 2). Therefore, it was possible that there were two independent UV repair systems active in $S$. cattleya spores during germination. To examine whether these systems were true host mediated repair systems affected by the inhibitors, a bacteriophage system was used. 


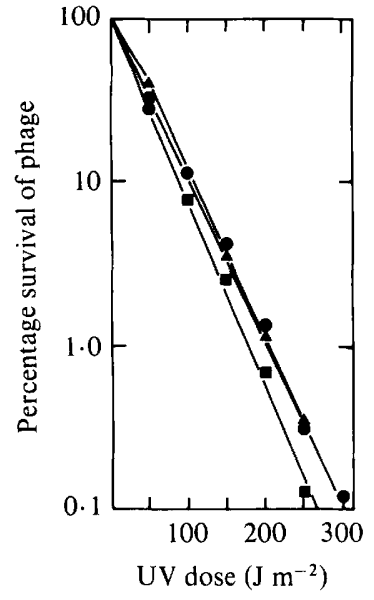

Fig. 4

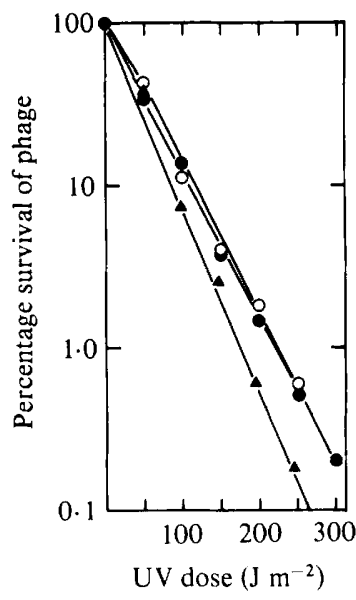

Fig. 5

Fig. 4. Effect of caffeine and sodium arsenite on the survival of UV irradiated actinophage VC11. $\boldsymbol{0}$, No inhibitor; $\boldsymbol{\Delta},+$ sodium arsenite; $\boldsymbol{\square},+$ caffeine.

Fig. 5. Effect of caffeine and sodium arsenite on the survival of UV irradiated actinophage VC11 plated on spores irradiated with UV $\left(50 \mathrm{~J} \mathrm{~m}^{-2}\right)$. No inhibitor; $\boldsymbol{\Delta}$, + caffeine; $\mathrm{O},+$ arsenite.

Study of UV irradiation repair in spores of $S$. cattleya using the virulent bacteriophage VC11

The use of a bacteriophage gives the opportunity to separate DNA damage from host repair systems and to determine whether these systems are constitutive or inducible. The bacteriophage is dependent on host enzymes for DNA replication and repair. Figure 3 shows that in the absence of UV irradiation of the host the survival of actinophage VC11 followed simple one hit kinetics. This would be expected in the absence of a repair system in the germinating spores. Irradiation of the host spores $\left(50 \mathrm{~J} \mathrm{~m}^{-2}\right)$ prior to plating increased phage survival by $100 \%$ or more and created a distinct shoulder to the survival curve. This is characteristic of the induction in the germinating spores of a system which can repair UV damage in the bacteriophage DNA. It was then necessary to study the effects of the two UV repair inhibitors on the survival of bacteriophage both with and without $U V$ induction of the spores.

Inhibition of bacteriophage repair by either caffeine or arsenite would be good evidence for an active repair system. In the presence of caffeine there was a reduction in the survival of VC11 (Fig. 4), indicating inhibition of a host cell repair system which acted on the phage. No such effect was observed with arsenite. This experimental methodology identified only one UV repair system in the germinating spores; the system was caffeine inhibitable and thus is analogous to 'error-free' repair.

The inducible system identified above may or may not be the same as the caffeine inhibitable system. Figure 5 shows the survival of VC11 with and without UV irradiation when grown on UV irradiated spores. Survival was lower in the presence of caffeine than in its absence. This lowered survival was similar to that obtained in the absence of UV irradiation of the spores, which shows that both the constitutive and the inducible system are caffeine inhibited. It does not show that they are one and the same. No such effect was observed with arsenite and so the presence of an arsenite inhibitable system, postulated from the spore survival results, was not confirmed.

\section{Effect of caffeine and arsenite on the genetic instability of $S$. cattleya}

If UV repair systems are involved in genetic instability in $S$. cattleya, the presence of inhibitors of UV repair should affect the instability. Figure 6 shows the frequency of 


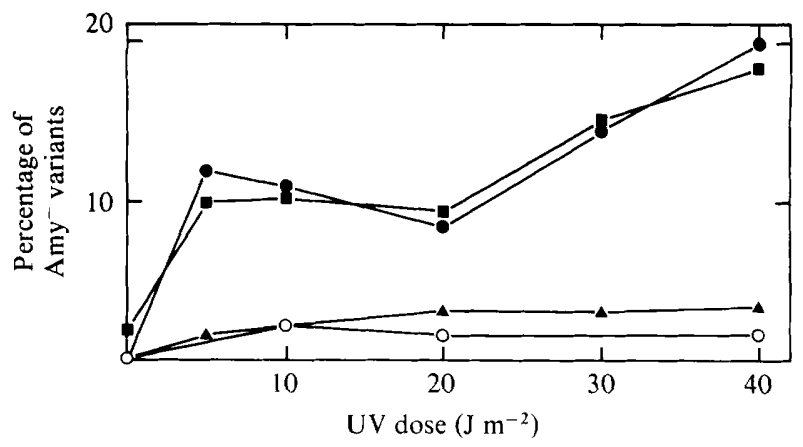

Fig. 6. Effect of caffeine and sodium arsenite on genetic instability in Streptomyces cattleya. No inhibitor; $\boldsymbol{\Delta},+$ caffeine; $\boldsymbol{\square},+$ sodium arsenite; $O$, caffeine and sodium arsenite.

$\mathrm{Amy}^{-} \mathrm{Arg}^{-}$variants of $S$. cattleya at increasing doses of UV and in the presence and absence of the two inhibitors of UV repair. Caffeine inhibited the induction of these variants and thus must have been inhibiting the system responsible for their induction. It should also be noted that arsenite induced a sixfold increase in instability of this type in the absence of UV. These results suggest the involvement of systems in the induction events which are affected by these chemicals.

\section{Effect of ${ }^{32} P$ on the genetic instability of $S$. cattleya}

Single strand breaks, which are caused by ${ }^{32} \mathrm{P}$ at $-70{ }^{\circ} \mathrm{C}$, are one of the most important events in the induction of 'error-free' repair. Thus if an 'error-free' type repair system is active in inducing genetic instability, ${ }^{32} \mathrm{P}$ should affect instability and perhaps interact with the inhibitors. In the presence of ${ }^{32} \mathrm{P}$ the frequency of $\mathrm{Amy}^{-} \mathrm{Arg}^{-}$variants increased 17 -fold from $0.5 \%$ in the absence of ${ }^{32} \mathrm{P}$ to $8.4 \%$ in its presence. Caffeine failed to inhibit this effect.

\section{DISCUSSION}

Figures 3 and 4 show that there is a constitutive caffeine inhibitable host mediated UV repair system. This system could be similar to the 'error-free' repair systems in $E$. coli. Caffeine also inhibited a UV induced repair system. As yet it is not possible to say whether there are two separate systems or whether they are constitutive and induced components of the same system. As it is not possible to compare directly the repair systems in species as diverse as $E$. coli and $S$. cattleya, the features of this system when studied further may be very different from $E$. coli.

Although there was preliminary evidence for an arsenite inhibitable DNA repair system in $S$. cattleya (Fig. 2), this was not confirmed by bacteriophage studies (Figs 4, 5 and 6). An arsenite inhibitable repair system could be of a type similar to the "error-prone' repair in $E$. coli and could involve an 'SOS-like' response. In $E$. coli, the SOS response only persists for approximately 20 min (Rossman et al., 1975). The time between UV irradiation of the $S$. cattleya spores and germination of the spores which allowed phage attachment was between 4 and $6 \mathrm{~h}$. Thus it is likely that any 'SOS-like' response would have decayed before phage attachment. Experiments involving irradiation of germinating spores and sequential addition of UV irradiated bacteriophage could detect such a system.

The effect of caffeine on the induction of genetic instability in S. cattleya showed that a component of the repair system or systems inhibited by this chemical was responsible for the genetic instability. The increase in genetic instability in the presence of arsenite supported the suggestion that there may be an arsenite inhibited repair system, not detected here using actinophage $\mathrm{VCl1}$, which is responsible for repairing the damage which induces the genetic events. 
The results of experiments with ${ }^{32} \mathrm{P}$-labelled orthophosphate confirmed that single strand breaks are implicated in the induction of genetic instability in $S$. cattleya spores. The effect of ${ }^{32} \mathrm{P}$ was not inhibited by caffeine, suggesting that the breaks are the primary event causing instability and not an effect of the repair system.

The interaction of UV damage and UV repair systems in genetic instability in S. cattleya gives the first insight into the biochemical basis of this phenomenon in Streptomyces. It may allow the selection of specific mutations affecting UV repair, which would in turn affect genetic instability. This could give rise to methods of reducing instability in industrially important Streptomyces.

\section{REFERENCES}

Flett, F., Wotten, S. F. \& Kirby, R. (1979). A common host-specificity in the restriction and modification of a bacteriophage by three distinct Streptomyces species. Journal of General Microbiology $110,465-467$.

KIRBY, R. \& LEWIS, E. (1981). Unstable genetic elements affecting streptomycin resistance in the streptomycin-producing organisms Streptomyces griseus NCIB 8506 and Streptomyces bikiniensis ISP 5235. Journal of General Microbiology 122, 351-355.

Martin, J. F., Luengo, J. M., Revilla, G. \& Villanueva, J. R. (1979). Biochemical genetics of the beta-lactam antibiotic biosynthesis. In $3 r d$ International Symposium on the Genetics of Industrial Microorganisms, pp. 83-89. Edited by O. K. Sebek \& A. I. Laskin. Washington, D. C.: American Society for Microbiology.
Pogell, B. M. (1979). Regulation of aerial mycelium formation in Streptomycetes. In 3rd International Symposium on the Genetics of Industrial Microorganisms, pp. 218-224. Edited by O. K. Sebek \& A. I. Laskin. Washington, D. C.: American Society for Microbiology.

Rossman, T., Meyn, M. S. \& Troll, W. (1975). Effect of sodium arsenite on the survival of UV-irradiated Escherichia coli: inhibition of a recA-dependent function. Mutation Research 30, 157-162.

Rothman, R. H. (1980). Dimer exision in Escherichia coli in the presence of caffeine. Journal of Bacteriology 143, 520-524.

SchumanN, J. P., Jones, D. J. \& Woods, D. R. (1982). UV light induction of proteins in Bacteroides fragilis under anaerobic conditions. Journal of Bacteriology 151, 44-47. 